\title{
Understanding stakeholders' perspectives on implementing deprescribing for older people living in long-term residential care homes: the STOPPING study protocol
}

Krystal Warmoth ${ }^{1}$, Jo Day² ${ }^{*}$ D, Emma Cockcroft ${ }^{2}$, Donald Nigel Reed ${ }^{3}$, Lucy Pollock ${ }^{4}$, George Coxon ${ }^{5}$, Julia Heneker ${ }^{6}$, Bridget Walton ${ }^{7}$ and Ken Stein ${ }^{2}$

\begin{abstract}
Background: Older people with multimorbidity often experience polypharmacy. Taking multiple medicines can be beneficial; however, some older adults are prescribed multiple medicines when they are unlikely to improve clinical outcomes and may lead to harm. Deprescribing means reducing or stopping prescription medicines which may no longer be providing benefit. While appropriate deprescribing may usually be safely undertaken, there is a lack of guidance about how to implement it in practice settings such as care homes. Implementing deprescribing in care homes is often challenging, due to differing concerns of residents, staff, clinicians, friends/family members and carers along with differences in care home structures. The STOPPING study will support the development of better deprescribing practice in care homes, considering different views and environments. This paper aims to introduce the research protocol.
\end{abstract}

Methods: We will use qualitative approaches informed by the widely accepted Consolidated Framework for Implementation Research (CFIR) to aid analysis. To understand the barriers, facilitators, and contextual factors influencing deprescribing in care homes, we will employ individual interviews with care home residents and family members, focus groups with care home staff and healthcare professionals, and observations from care homes. Then, we will examine acceptability, feasibility, and suitability of existing deprescribing approaches using cognitive interviews with care home staff and healthcare professionals. Lastly, we will use narrative synthesis to integrate findings and develop guidance for implementing a deprescribing approach for care homes.

Discussion: This research will support the development of implementable approaches to deprescribing in care homes. The insights from this project will be shared with various stakeholders: care home residents, staff, pharmacists, general practitioners, nurses, and other health professionals, carers, researchers, and the public. This work will support deprescribing to be implemented effectively in care homes to benefit residents and the wider health economy.

Keywords: Medicine optimisation, Qualitative, Older adults, Polypharmacy, Care homes, Implementation

\footnotetext{
* Correspondence: J.K.Day@exeter.ac.uk

${ }^{2}$ College of Medicine and Health, University of Exeter, St Luke's Campus, Heavitree Road, Exeter EX1 2LU, UK

Full list of author information is available at the end of the article
}

(c) The Author(s). 2020 Open Access This article is licensed under a Creative Commons Attribution 4.0 International License, which permits use, sharing, adaptation, distribution and reproduction in any medium or format, as long as you give appropriate credit to the original author(s) and the source, provide a link to the Creative Commons licence, and indicate if changes were made. The images or other third party material in this article are included in the article's Creative Commons licence, unless indicated otherwise in a credit line to the material. If material is not included in the article's Creative Commons licence and your intended use is not permitted by statutory regulation or exceeds the permitted use, you will need to obtain permission directly from the copyright holder. To view a copy of this licence, visit http://creativecommons.org/licenses/by/4.0/ The Creative Commons Public Domain Dedication waiver (http://creativecommons.org/publicdomain/zero/1.0/) applies to the data made available in this article, unless otherwise stated in a credit line to the data. 


\section{Contributions to the literature}

- Research indicates that deprescribing (the reducing or stopping some prescription medicines which may no longer be providing benefit) is generally safe, but how to best implement it in real-life settings, such as care homes, is unknown.

- Implementing deprescribing in care homes can be challenging due to the different concerns of residents, staff, clinicians, family members, and carers and variability in care home structures.

- The current protocol outlines a project that will inform the development of an implementable, context-sensitive deprescribing approach in care homes.

\section{Background}

Polypharmacy (the concurrent use of multiple medicines) is a common experience for older adults. Four out of five people aged over 75 are prescribed medicines, with over a third taking 6 or more [1]. In UK care homes, the prevalence of polypharmacy is widespread and increasing [2]. Almost one half of care home residents are exposed to potentially inappropriate medicines, which increases over time [3]. Care home staff, residents, and family members stress the high prevalence, fears about the health and safety consequences, and daily burden of polypharmacy at care homes [4]. To mitigate the harms of polypharmacy [5-7], National Institute for Health and Care Excellence (NICE) commends deprescribing in some situations, including as part of the holistic review of a person with multimorbidity [8]. Deprescribing is defined as the process of withdrawing inappropriate medicine and reducing or stopping medicines which may no longer be providing benefit, supervised by a healthcare professional' [9]. While appropriate deprescribing is usually commended and may be cautiously undertaken to good effect [10, 11 , there is a lack of guidance both about how to implement it safely and appropriately [9].

Taking multiple medicines can be beneficial; however, some older adults continue to be prescribed multiple medicines when they are unlikely to improve clinical outcomes and/or lead to harm. Polypharmacy is associated with an increased risk of impaired cognition, falls, morbidity, disability, frailty, and death [5-7], and adverse drug reactions are associated with $11 \%$ of hospital admissions in people over 65 years old [10]. These problems are ubiquitous in care homes, where between 50 and $70 \%$ of residents are exposed to one or more medication errors $[12,13]$. With an ageing population, these figures are expected to rise, and medicine optimisation is a priority for healthcare services [14].
Withdrawing certain medication classes (e.g. psychotropic drugs and proton-pump inhibitors) may reduce adverse events (such as falls) and improve wellbeing [15]. There are some limitations of previous deprescribing studies that need to be considered, namely unsuccessful intervention implementation and lack of examination of the patient-important outcomes (e.g. treatment burden). Overall, little is known about how to best implement deprescribing in real-world conditions, with many studies overlooking context and stakeholder views [16]. Implementation aspects of existing deprescribing approaches are typically not well-described and poorly understood [11, 17]. Moreover, deprescribing studies, which are mostly quantitative, measure the number of prescribed medicines reduced, and many do not consider the overall appropriateness of prescribing or clinically important outcomes (older adults' health) [11]. Deprescribing faces challenges related to poor implementation and considerable differences in reported acceptance rates among healthcare professionals $[18,19]$ and patients [20, 21]. Variable acceptability subsequently leads to suboptimal deprescribing and is a mechanism that the proposed work will address, by investigating views about existing deprescribing approaches.

Previous work has explored generic barriers and facilitators to deprescribing [22-31]. Most of these studies have only focused on the perspectives of a single stakeholder group, such as general practitioners [23-25], geriatricians [26], and patients [28, 30, 32]. They have not combined the different stakeholder perspectives or examined views about specific approaches. Only one study compared differing views [22] and proposed that the greatest implementation barrier to deprescribing is the differing expectations and perceptions of stakeholders. Different stakeholders will have different drivers and concerns (e.g. the family may pressure care home practitioners and health professionals to 'do more' and continue prescribing). Furthermore, there is a lack of resident perspectives in research studies because they can be the stakeholders with the weakest voice and are often underrepresented. To address this, we will gather and compare perspectives from various stakeholders in care homes (residents, family members, staff, general practitioners, and other healthcare professionals). Without such perspectives, a better understanding cannot be developed to inform intervention development.

The challenge of implementing deprescribing in practice is that it is complex, involves multiple stakeholders, and requires patient-centred and multidisciplinary approaches $[17,33]$. There are numerous interrelated and sometimes conflicting factors influencing implementation, especially with time-constraints and heavy workloads, and poor understanding of environmental context is suggested as the greatest barrier [34]. Differences in care home structures 
and functions along with the broader healthcare systems need to be considered, as they are likely to be important factors influencing implementation [35]. There is a great deal of variability in how care homes function, and this may be key in terms of how an intervention may or may not work. Context may be a barrier that impacts other factors (e.g. motivation, decision-making, relationships) [34]. Eliciting the diverse stakeholder perspectives and viewing these as aspects of the 'bigger picture' of the organisation will provide insights into the dynamics and interactions that may shape the success of deprescribing. Ways to operationalise implementation considerations as part of intervention development remain generally vague. There is a need to further understand how contextual factors that facilitate or hinder deprescribing that is informed by theories, models, and frameworks about implementation to inform intervention development.

\section{Study aims}

The overall aim is to inform the development of a deprescribing approach that can be implemented in care homes. By comparing different stakeholders' perspectives and utilising a framework from implementation science, we will investigate the implementation of deprescribing in care homes.

The specific objectives are to:

1. Identify the barriers, facilitators, and contextual factors influencing deprescribing in two contrasting types of care homes (independently owned and part of a larger organisation).
2. Evaluate the perceived acceptability, feasibility, and suitability of existing deprescribing approaches.

3. Generate guidance for developing a deprescribing approach for care homes.

\section{Method}

\section{Study design}

We will use qualitative inquiry [36] and adopt a pragmatic approach in separate work packages using diverse methodological approaches to achieve our objectives (see Fig. 1). Work package 1 will include interviews with residents and their family members/friends about their experiences, focus groups with staff and healthcare professionals about their experiences, and observations at care homes to identify the key barriers, facilitators, and contextual factors from various stakeholders and different care homes (objective 1). Work package 2 includes cognitive interviews with care home staff and healthcare professionals about current deprescribing approaches to evaluate the perceived acceptability, feasibility, and suitability of these approaches (objective 2). Explicit feedback and rich data on existing deprescribing interventions will be gathered. Work package 3 will synthesise the findings of Work packages 1 and 2 to produce an early version of a deprescribing approach which is suitable for care homes (objective 3).

\section{Study setting and context}

Data collection will be conducted at six care home sites of two different care home providers. These providers were approached to partner for this research as they represent contrasting models of care home provision.

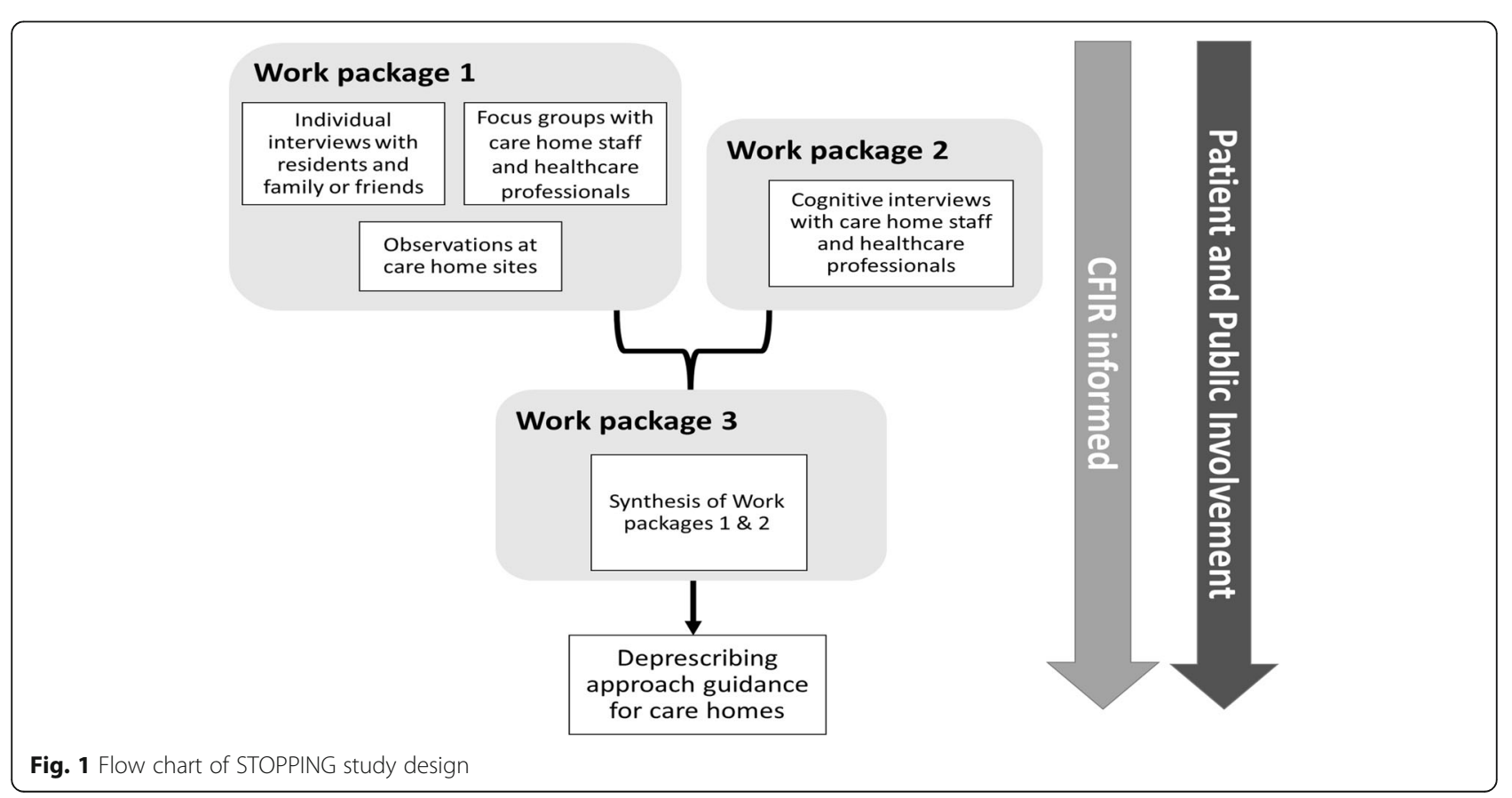


That is, one provider is a smaller independently owned organisation consisting of two residential care home sites and a large organisation with 27 care homes in several locations in the South of England. Care home sites included in this study were chosen in partnership between the research team and senior care home staff and selected as they mainly provided residential care (i.e. personal care without nursing) for older adults (over 65 years old).

\section{Participants}

In this project, we will work with four groups of participants: care home residents, residents' family and friends, and care home staff and healthcare professionals (e.g. general practitioners, nurses, and pharmacists). Purposive sampling will be used to select and recruit a wide range of experiences from the participants. Initially, the eligibility criteria will be applied to recruit participants for the different participant groups. We will then iteratively recruit participants to capture meaningful experiences within the sample.

The eligibility criteria for care home residents taking part in the research are:

- Being a resident of the participating care home, age 65 and over

- Taking multiple medications or experience of polypharmacy

- Having the ability to converse in English without an interpreter or professional assistance

- Having the absence of serious cognitive impairment, as identified by the care home staff or healthcare profession

- Having the capacity to consent to participation

Residents with severe cognitive impairment which inhibits consent will be excluded from being interviewed, but their family members and carers will be included, as this group are particularly at risk of overall drug burden and often benefit from deprescribing interventions.

The eligibility criteria for care home residents' family member or friend taking part in the study are being a family member or friend of a resident taking multiple medications at the care home participating in the study and having the ability to converse in English without an interpreter or professional assistance.

The eligibility criteria for care home staff and healthcare professionals participating in the focus groups and cognitive interviews will be the same. They must be currently working directly with older adults with polypharmacy and/or in the care home setting and ability to converse in English without an interpreter or professional assistance.

\section{Ethical considerations}

Ethical approval to conduct the study was given by the Social Care Research Ethics Committee (19/IEC08/ 0058). Participants will be informed both verbally and in writing about the aims, methods, procedures, and measures performed during the study. They will be also informed about ethical issues such as confidentiality, their right to ask any questions during the study, and their right to withdraw at any time without penalty. To ensure that all participants have received proper information about the study and have agreed to participate, participants will be asked to sign a written consent form.

For the care home observations, some residents without capacity may be observed. For these residents, consent of behalf of these residents will be obtained from a designated personal consultee (a family member or friend) who will be identified by the senior care home staff. Personal consultees will receive the same written information as would have been provided to their resident. If the resident does not have a family member or close friend who is able or willing to act as a personal consultee, then a nominated consultee will be appointed.

We will not encourage any participants to stop or reduce their medicines or change their medicine-taking behaviour. If they wish to change their medications as a result of the discussion with the researcher, we will let the care home staff know so that the resident and the care home team can discuss this with their general practitioner and any other relevant healthcare professionals and advise them not to change anything until they have consulted a healthcare professional.

If it is observed that a resident participating in the research is getting upset or tired from taking part, we will stop the observations or interview. The researcher will inform the care home staff what happened so appropriate steps can be taken.

Patient and public involvement and partnership approach A patient and healthcare professional partnership underpins this project. We have worked with care home residents and carers to develop the research, design this protocol, and prepare study materials. A patient/carer representative and a patient involvement facilitator are on the research team and will work together ensure full integration of patient involvement through the study. A group of care home residents will contribute to the interpretation of the study findings and development of dissemination materials and activities.

\section{Data collection}

Data collection will continue until data saturation occurs, meaning that no new ideas are being generated. The sample size was determined using previous 
literature on qualitative methods and purposeful sampling to select and recruit a wide range of experiences [37].

\section{Work package 1}

To identify the barriers, facilitators, and contextual factors to deprescribing in care homes (objective 1), we will conduct individual interviews, focus groups, and observations in this work package.

We will conduct semi-structured individual interviews with care home residents and family members from 6 different care home sites to elicit their perceptions, problems, and experiences regarding multiple medicines and deprescribing (i.e. reducing or stopping medicines). Resident and family member interviews will be conducted separately so that participants can speak openly about their views. We will meet in a private room within the care home to accommodate functional impairments, mobility issues, and frailty and for resident safety. Based on literature $[22,28,30,32]$ and CFIR, topics of discussion will include practices of medicine management in care homes, the lived experience of taking multiple medicines, and attitudes and beliefs towards deprescribing. However, we will allow for flexibility in topics to pursue issues in more depth as they emerged from the interviews. We expect the interviews will last approximately an hour. Interviews will be audio-recorded, and then, they will be transcribed verbatim for analysis. Reflective notes will be taken after the interview.

Two focus groups will be conducted separately with both care home staff and healthcare professionals [38]. Focus groups are used to analyse the specific nature of the problem and determine who is involved or affected by the problem and how the problem is perceived from different perspectives [39]. Each focus group will be attended by two members of the research team (one to facilitate discussion and one to take notes of the discussion). Before the start of the focus group, the facilitator will explain their role and the ground rules for the discussion. It will be stressed that the discussion is confidential and every opinion will be respected to encourage open discussion and the group members feel comfortable. Topics will be oriented to deprescribing in practice, and the topic guide will be informed by CFIR and previous literature [23-26, 29, 40, 41]. Discussions will focus on their views and experiences with deprescribing; structures and relationships between general practitioners, other healthcare professionals, staff, and residents; and the deprescribing practices already in place. This data will provide the perspectives of clinicians and other health providers on their priorities for the implementation (or non-implementation) of deprescribing It will also identify the perceived barriers, facilitators, and contextual factors that they perceive as relevant. Responses will help ensure that findings are translated into meaningful recommendations for changes in approaches to deprescribing in care homes. Conflicting views and interactions between stakeholders and sites will be documented. We expect the discussion will last approximately an hour.

Observations will be conducted at each of the 6 different care home sites ( 2 from the smaller provider and 4 from the larger provider). To start, detailed accounts of the existing procedures and policies regarding medicine management and monitoring for each site will be collected. Care home staff will be asked to demonstrate and tell the researcher about medicine routines and administration. The researcher will ask staff to talk them through medicine-taking practices and identify what informs them. Then, informed by the practices at each site, observations of the different practices and procedures will be conducted (e.g. medication reviews, medicine administration, or relevant training). Over 2-3 weeks, approximately 10 observations (lasting approximately $30 \mathrm{~min}$ to $1 \mathrm{~h}$ ) will be conducted at varying times at each care home site to capture a range of behaviours and practices. Observations will be conducted in communal areas of the care homes and only in private areas (e.g. resident's bedroom) if the researcher is invited by the resident. Observations will be recorded using anonymised fieldnotes and a reflective diary. This method allows the opportunity to see the daily routines and experiences in context-not retrospectively-which may provide differing findings.

\section{Work package 2}

In Work package 2, explicit views about existing deprescribing approaches will be investigated, building on a better understanding of contextual factors influencing deprescribing gained from the previous work package. Cognitive interviewing is a widely used method for developing and evaluating questionnaires; it identifies thought processes behind decisions [42]. It has not been used in the evaluation of deprescribing approaches and may generate fresh insight. Cognitive interviewing uses two techniques: the 'think aloud interview' where participant thought processes are described and 'verbal probing' where the investigator asks the participant direct questions, followed by questions to explore participant motivations and understanding. Probes and questions will be informed by CFIR. The method will allow for feedback and criticisms of five existing deprescribing approaches (e.g. Screening Tool of Older Person's Prescriptions or STOPP [43]), recommended by clinical and pharmaceutical colleagues and NICE guidelines. Responses will help determine whether the existing approaches are suitable in care homes and identify key components that may be missing, inappropriate, or 
crucial for care homes. Findings will specify explicit limitations, facilitators and possible modifications for existing deprescribing approaches. We expect the interview will last approximately an hour. Responses will be audiorecorded and transcribed verbatim.

\section{Data analysis}

For Work package 1, the framework approach [44] will be employed for thematic analysis of the interview and focus group responses as well as the observation notes. In this approach, data is sifted, charted, and organised using a coding framework. This will be informed by both CFIR and unexpected themes, allowing for comparing across stakeholder groups and care homes. Any disagreements among the research team in the analysis process will be discussed and resolved. Preliminary findings will be discussed with PPI and project advisory groups to ensure that our interpretations are clinically relevant and fit for purpose. Lay summaries of the preliminary findings will be developed and sent to participants for comment.

For Work package 2, the analysis will begin with interview transcripts being subject to the first cycle of evaluative coding, assigning a judgement about the merit or significance of the approach [45]. We will note the presence or absence of an attribute (e.g. shared decisionmaking) and how positively or negatively it was evaluated. Second cycle coding will extend the mapping of variance across the dataset, enabled by within-case and cross-case analysis [45]. Findings from this analysis will determine the limitations and suitability of specific deprescribing approaches for different types of care homes. Any discrepancies among the research team will be discussed and resolved. The findings will then be mapped onto CFIR and compared with its constructs and themes. Summaries of the findings will be developed and sent to participants for comment.

The final work package will combine Work packages 1 and 2 data. Using a narrative synthesis approach [46], we will synthesise the overarching findings and identified themes and interpret them, with the aid of CFIR and PPI group input, to create detailed guidance for a deprescribing approach (objective 3). This synthesis will identify what barriers, facilitators, and contextual factors need to be addressed in the development of a deprescribing approach for care homes. We will compare findings from the individual interview and focus groups with cognitive interviews and ascertain any discrepancies between different stakeholders and types of care homes. We will determine the areas for improvement of the implementation of existing deprescribing approaches.

First, the data will be aggregated for similar themes; then, it will be configured for thematically diverse findings. Facilitators and barriers to deprescribing will be listed, using established techniques (e.g. tabulation and vote counting) [46]. We will consider how and why barriers, enablers, and contextual factors operate from emerging relationships via conceptual mapping and triangulation. This theoretical understanding will inform guidance for further intervention development.

All data will be managed with NVivo 12 software which can manage the diverse types data collected (i.e. fieldnotes, focus group, and interview transcripts) [47], and NVivo's framework matrix provides a way to summarise source materials by case (e.g. for each care home) and theme (e.g. CFIR construct).

\section{Discussion}

The most effective way to implement deprescribing in care home setting is unknown [48, 49]. This research will inform the development of an implementable, context-sensitive deprescribing approach in care homes (objective 3), by incorporating key stakeholder views and CFIR domains. The likelihood of success of this deprescribing approach will be improved by developing a comprehensive understanding of context, barriers, and facilitators to implementation (objective 1) and appraising the implementation of existing deprescribing approaches (objective 2). Furthermore, by comparing contrasting care home organisations (independently owned and part of a larger organisation), we can suggest strategies for tailoring to these specific organisations. Successfully implementing deprescribing in care homes could potentially enhance the residents' wellbeing by achieving a balance between the benefits and harm of polypharmacy, reducing treatment burden [4], and maintaining control of chronic conditions.

One of the strengths of this work is that we will be considering the contextual factors that influence the implementation of any deprescribing approach at the very outset of intervention development. This understanding will provide crucial information about acceptability and suitability for residents, carers, and healthcare professionals. Another strength is that we will be using a comprehensive, well-recognised implementation science framework. We will be drawing from CFIR to investigate how to implement deprescribing in real-world settings [16], specifically care homes [50]. Using this framework will help identify crucial influences, address barriers to deprescribing, and ensure that interventions are effective and sustainable $[49,51]$. An additional strength of the study is the collection of data through not only reflective interviews and focus groups but also collected in real-life settings (i.e. observations in the care homes). Observations will provide a better understanding of everyday practices and insight into experiences in different care homes than focus groups and interviews alone. Another notable strength of this research is the patient and 
public involvement and partnership approach. We have worked and will continue to work with care home residents to help ensure the project remains focused on patient benefit and includes views from all involved in deprescribing (family members, residents, etc.).

A potential limitation of the study is the low participant numbers. We will iteratively recruit to maximise any meaningful experiences within the sample. Rich data will be collected from each participant, and the findings will be combined across work packages to achieve a comprehensive understanding. Another potential limitation is excluding participants who are unable to converse in English and show signs of severe cognitive impairment. These individuals may have different experiences and perspectives, limiting the generalisability of study findings. To address this limitation, we have included care home observations and interviews with family and friends of these older adults to try to capture these experiences and views.

Ageing is one of the greatest social and economic challenges for society as the ageing population is growing and living longer. The insights from this project can inform the development of a deprescribing approach that is contextually grounded and implementable in care homes. This knowledge will be shared with various stakeholders: care home residents, staff, pharmacists, general practitioners, nurses, and other health professionals, carers, policymakers, researchers, and the public. This work will allow deprescribing to be implemented safely and effectively in care homes to benefit residents and the wider health economy.

\section{Abbreviations \\ STOPPING: Understanding stakeholders' perspectives on implementing deprescribing in care homes; CFIR: Consolidated Framework for Implementation Research; PPI: Patient and public involvement; NICE: National Institute for Health and Care Excellence; STOPP: Screening Tool of Older Person's Prescriptions; NHS: National Health Service; UK: United Kingdom}

\section{Acknowledgements}

We greatly appreciate the feedback from care home staff and residents who attended the PPI events that played an integral role in guiding our project. We gratefully acknowledge the input of the care home staff and residents from Somerset Care Ltd. and Pottles Court and Summercourt care homes. We also acknowledge the input of the Project Advisory Group members, Laura Picton, Professor Jose Valderas Martinez, Dr Laura Sims, Professor Nicky Britten, Dr lain Lang, and Charlene Ronquillo.

\section{Authors' contributions}

KW led the drafting of this manuscript. KW, JD, EC, KS, DNR, LP, GC, and JH co-applied for funding. BW critically revised and drafted the manuscript. All authors contributed to the manuscript and reviewed and approved the final version of the paper.

\section{Funding}

This project is funded by the National Institute for Health Research (Research for Patient Benefit, Understanding stakeholders' perspectives on implementing deprescribing in care homes (STOPPING), PB-PG-0418-20026). This is an independent research supported by the National Institute for Health Research (NIHR) Applied Research Collaboration for the East of England and South West Peninsula.
The views expressed in this publication are those of the authors and not necessarily those of the NHS, NIHR, or the Department of Health and Social Care.

\section{Availability of data and materials}

Materials described in this paper pertain to the study protocol only and there are no raw data reported.

\section{Ethics approval and consent to participate}

Ethical approval to conduct the study was given by Social Care Research Ethics Committee (19/IEC08/0058). Participants are required to sign a consent form to indicate their willingness to participate. Voluntary participation and the right to ask any questions and to decline participation at any time will be emphasised during the data collection.

\section{Consent for publication}

Not applicable

\section{Competing interests}

The authors declare that they have no competing interests.

\section{Author details}

${ }^{1}$ Centre for Research in Public Health and Community Care, University of Hertfordshire, Hatfield, UK. ${ }^{2}$ College of Medicine and Health, University of Exeter, St Luke's Campus, Heavitree Road, Exeter EX1 2LU, UK. ${ }^{3}$ Peninsula Public Involvement Group, National Institute for Health Research Applied Research Collaboration South West Peninsula, Exeter, UK. ${ }^{4}$ Musgrove Park Hospital, Somerset NHS Foundation Trust, Taunton, UK. ${ }^{5}$ Pottles Court and Summercourt Care Homes, Exminster and Teignmouth, UK. ${ }^{6}$ Somerset Care Ltd., Taunton, UK. ${ }^{7}$ Royal Devon and Exeter NHS Foundation Trust, Exeter, UK.

Received: 18 August 2020 Accepted: 21 August 2020

Published online: 03 September 2020

\section{References}

1. Moody A, Mindell J, Faulding S, Neave A. Health Survey for England 2016: Prescribed medicines. In: Health and Social Care Information Centre, editor: NHS Digital,; 2017

2. Duerden M, Avery T, Payne R. Polypharmacy and medicines optimisation: making it safe and sound. London: The King's Fund; 2013.

3. Morin L, Laroche M-L, Texier G, Johnell K. Prevalence of potentially inappropriate medication use in older adults living in nursing homes: a systematic review. J Am Med Dir Assoc. 2016;17(9):e861-2 e9.

4. Gnjidic D, Tinetti M, Allore HG. Assessing medication burden and polypharmacy: finding the perfect measure. Expert Rev Clin Pharmacol. 2017;10(4):345-7.

5. Turner JP, Jamsen KM, Shakib S, Singhal N, Prowse R, Bell JS. Polypharmacy cut-points in older people with cancer: how many medications are too many? Support Care Cancer. 2016;24(4):1831-40.

6. Jyrkka J, Enlund H, Korhonen MJ, Sulkava R, Hartikainen S. Polypharmacy status as an indicator of mortality in an elderly population. Drugs Aging 2009:26(12):1039-48.

7. Gnjidic D, Hilmer SN, Blyth FM, Naganathan V, Waite L, Seibel MJ, et al. Polypharmacy cutoff and outcomes: five or more medicines were used to identify community-dwelling older men at risk of different adverse outcomes. J Clin Epidemiol. 2012;65(9):989-95

8. National Institute for Health and Care Excellence (NICE). Multimorbidity: clinical assessment and management (NG56). 2016.

9. Reeve E, Gnjidic D, Long J, Hilmer S. A systematic review of the emerging definition of 'deprescribing' with network analysis: implications for future research and clinical practice. Br J Clin Pharmacol. 2015;80(6):1254-68.

10. Kongkaew C, Noyce PR, Ashcroft DM. Hospital admissions associated with adverse drug reactions: a systematic review of prospective observational studies. Ann Pharmacother. 2008;42(7-8):1017-25.

11. Cooper JA, Cadogan CA, Patterson SM, Kerse N, Bradley MC, Ryan C, et al. Interventions to improve the appropriate use of polypharmacy in older people: a Cochrane systematic review. BMJ Open. 2015;5(12).

12. Barber ND, Alldred DP, Raynor DK, Dickinson R, Garfield S, Jesson B, et al. Care homes' use of medicines study: prevalence, causes and potential harm of medication errors in care homes for older people. Qual Saf Health Care. 2009;18(5):341. 
13. Szczepura A, Wild D, Nelson S. Medication administration errors for older people in long-term residential care. BMC Geriatr. 2011;11(1):82.

14. Naylor C, Imison C, Addicott R, Buck D, Goodwin N, Harrison T, et al. Transforming our helath care system: ten priorities for commissioners: The King's Fund; 2015.

15. van der Cammen TJM, Rajkumar C, Onder G, Sterke CS, Petrovic M. Drug cessation in complex older adults: time for action. Age Ageing. 2014;43(1):20-5.

16. Reeve $\mathrm{E}$, Thompson W, Farrell B. Deprescribing: a narrative review of the evidence and practical recommendations for recognizing opportunities and taking action. Eur J Internal Med. 2017;38:3-11.

17. Page AT, Clifford RM, Potter K, Schwartz D, Etherton-Beer CD. The feasibility and effect of deprescribing in older adults on mortality and health: a systematic review and meta-analysis. $\mathrm{Br} J$ Clin Pharmacol. 2016:82(3):583-623.

18. Brulhart MI, Wermeille JP. Multidisciplinary medication review: evaluation of a pharmaceutical care model for nursing homes. Int J Clin Pharm. 2011; 33(3):549-57.

19. Kroenke L, Pinholt EM. Reducing polypharmacy in the elderly. J Am Geriatr Soc. 1990;38(1):31-6.

20. Williams ME, Pulliam CC, Hunter R, Johnson TM, Owens JE, Kincaid J, et al. The short-term effect of interdisciplinary medication review on function and cost in ambulatory elderly people. J Am Geriatr Soc. 2004:52(1):93-8.

21. Garfinkel D, Mangin D. Feasibility study of a systematic approach for discontinuation of multiple medications in older adults: addressing polypharmacy. Arch Intern Med. 2010;170(18):1648-54.

22. Palagyi A, Keay L, Harper J, Potter J, Lindley RI. Barricades and brickwalls-a qualitative study exploring perceptions of medication use and deprescribing in long-term care. BMC Geriatr. 2016;16(1):15.

23. Schuling J, Gebben H, LJG V, Haaijer-Ruskamp FM. Deprescribing medication in very elderly patients with multimorbidity: the view of Dutch GPs. A qualitative study. BMC Fam Pract. 2012;13(1):56.

24. Moen J, Norrgård S, Antonov K, Nilsson JLG, Ring L. GPs' perceptions of multiple-medicine use in older patients. J Eval Clin Pract. 2010;16(1):69-75.

25. Anthierens S, Tansens A, Petrovic M, Christiaens T. Qualitative insights into general practitioners views on polypharmacy. BMC Fam Pract. 2010;11(1):65.

26. Ní Chróinín D, Ní Chróinín C, Beveridge A. Factors influencing deprescribing habits among geriatricians. Age Ageing. 2015:44(4):704-8.

27. Cullinan S, Hansen CR, Byrne S, O'mahony D, Kearney P, Sahm L. Challenges of deprescribing in the multimorbid patient. Eur J Hosp Pharm. 2017;24(1):43-6.

28. Linsky A, Simon SR, Bokhour B. Patient perceptions of proactive medication discontinuation. Patient Educ Couns. 2015;98(2):220-5.

29. Linsky A, Simon SR, Marcello TB, Bokhour B. Clinical provider perceptions of proactive medication discontinuation. Am J Manag Care. 2015;21(4):277-83.

30. Reeve E, Wiese MD, Hendrix I, Roberts MS, Shakib S. People's attitudes, beliefs, and experiences regarding polypharmacy and willingness to deprescribe. J Am Geriatr Soc. 2013;61(9):1508-14.

31. Reeve E, To J, Hendrix I, Shakib S, Roberts MS, Wiese MD. Patient barriers to and enablers of deprescribing: a systematic review. Drugs Aging. 2013; 30(10):793-807.

32. Kalogianis MJ, Wimmer BC, Turner JP, Tan ECK, Emery T, Robson L, et al. Are residents of aged care facilities willing to have their medications deprescribed? Res Soc Adm Pharm. 2016;12(5):784-8.

33. Gnjidic D, Le Couteur DG, Kouladjian L, Hilmer SN. Deprescribing trials: methods to reduce polypharmacy and the impact on prescribing and clinical outcomes. Clin Geriatr Med 2012;28(2):237-253.

34. Cadogan CA, Ryan C, Francis JJ, Gormley GJ, Passmore P, Kerse N, et al. Improving appropriate polypharmacy for older people in primary care: selecting components of an evidence-based intervention to target prescribing and dispensing. Implement Sci. 2015;10(1):161.

35. Competition and Markets Authority. Care homes market study London; 2017.

36. Duggleby W, Williams A. Methodological and epistemological considerations in utilizing qualitative inquiry to develop interventions. Qual Health Res. 2016;26(2):147-53.

37. Guest G, Bunce A, Johnson L. How many interviews are enough? An experiment with data saturation and variability. Field Methods. 2006 18(1):59-82.

38. Barbour R. Doing focus groups. 2nd ed. London: SAGE; 2018.

39. O'Cathain A, Thomas KJ, Drabble SJ, Rudolph A, Goode J, Hewison J. Maximising the value of combining qualitative research and randomised controlled trials in health research: the QUAlitative Research in Trials (QUART) study--a mixed methods study. Health Technol Assess. 2014;18(38).

40. Nixon MS, Vendelø MT. General practitioners' decisions about discontinuation of medication: an explorative study. J Health Organ Manag. 2016;30(4):565-80.

41. Nixon MS, Kousgaard MB. Organising medication discontinuation: a qualitative study exploring the views of general practitioners toward discontinuing statins. BMC Health Serv Res. 2016;16(1):226.

42. Willis GB. Cognitive interviewing: a tool for improving questionnaire design: Sage Publications; 2004

43. Gallagher P, Ryan C, Byrne S, Kennedy J, O'Mahony D. STOPP (Screening Tool of Older Person's Prescriptions) and START (Screening Tool to Alert doctors to Right Treatment). Consensus validation. Int J Clin Pharmacol Ther. 2008;46(2):72-83.

44. Ritchie J, Spencer L. The qualitative researcher's companion. 2002 2018/04/ 04. Thousand Oaks: SAGE Publications, Inc. Available from: http://methods. sagepub.com/book/the-qualitative-researchers-companion.

45. Miles MB, Huberman AM, Saldana J. Qualitative data analysis: a methods sourcebook. SAGE: Los Angeles; 2013

46. Popay J, Roberts H, Sowden A, Petticrew M, Arai L, Rodgers M, et al. Guidance on the conduct of narrative synthesis in systematic reviews: ESRC methods programme; 2006. p. 92.

47. QSR International Pty Ltd. NVivo qualitative data analysis software. 12 ed 2018.

48. Anderson K, Stowasser D, Freeman C, Scott I. Prescriber barriers and enablers to minimising potentially inappropriate medications in adults: a systematic review and thematic synthesis. BMJ Open. 2014:4(12):e006544.

49. Ronquillo C, Day J, Warmoth K, Britten N, Stein K, Lang I. An implementation science perspective on deprescribing. Public Policy Aging Rep. 2018;28(4):134-9

50. Alldred DP, Kennedy M, Hughes C, Chen TF, Miller P. Interventions to optimise prescribing for older people in care homes. Cochrane Database Syst Rev. 2016;2

51. Gnjidic D, Le Couteur DG, Hilmer SN. Discontinuing drug treatments. BMJ. 2014;349.

\section{Publisher's Note}

Springer Nature remains neutral with regard to jurisdictional claims in published maps and institutional affiliations.

Ready to submit your research? Choose BMC and benefit from:

- fast, convenient online submission

- thorough peer review by experienced researchers in your field

- rapid publication on acceptance

- support for research data, including large and complex data types

- gold Open Access which fosters wider collaboration and increased citations

- maximum visibility for your research: over $100 \mathrm{M}$ website views per year

At BMC, research is always in progress.

Learn more biomedcentral.com/submissions 